\title{
La comunicación como medio para la cohesión social en las familias tradicionales
}

\author{
Gonzalo Haro Álvarez \\ Facultad de Economía, Benemérita Universidad Autónoma de Puebla. \\ gonzalo.haro@correo.buap.mx \\ ORCID: https://orcid.org/0000-0002-8440-5966 \\ Milagros Bajo Ortega \\ Departamento de Ciencias Sociales y Humanidades, Universidad Autónoma de Occidente. \\ milagrosbajo44@gmail.com \\ ORCID: https://orcid.org/0000-0003-3127-7224 \\ Fecha de finalización: 31 de agosto de 2021 . \\ Recibido: 1 de septiembre de 2021 . \\ Aceptado: 25 de octubre de 2021 \\ DOI: https://doi.org/10.26422/aucom.2021.1002.har
}

\section{Resumen}

La comunicación es un elemento esencial en la conducta y comportamiento de los integrantes de una familia, pero ¿será suficiente para llegar a la cohesión familiar?, ¿qué explicaría la unión en las familias? El objetivo del trabajo es contextualizar el entorno de los integrantes de la familia tradicional a partir de sus necesidades tangibles e intangibles para una mejor comunicación y unión familiar basados en el vínculo social, la confianza, los valores compartidos y el sentido de pertenencia. La metodología del estudio es cuantitativa, de corte transversal y descriptiva. Para ello, se diseñaron tres cuestionarios para administrar a cada uno de los integrantes (papá, mamá e hijo) de familias tradicionales de la comunidad Los Ángeles del Triunfo, Guasave, Sinaloa (México). Los resultados arrojan que la mamá mantiene mayor comunicación con su esposo e hijos, el papá presentó menor acercamiento con su esposa e hijos y estos se comunican más con su mamá. Asimismo, se concluye que comunicación, confianza y tolerancia son imprescindibles en la cohesión de las familias tradicionales.

Palabras clave: familia tradicional, cohesión social, comunicación, necesidades, contexto familiar.

\section{Communication as a means for social cohesion in traditional families} Abstract

Communication is an essential part of the conduct and behavior of family members. But is it enough to achieve cohesion within the family unit? And what brings a family together? Our goal is to contextualize the environment of traditional family members, looking at their tangible and intangible needs for better communication and family unity based on social ties, trust, shared values, and a sense of belonging. Our study methodology is quantitative, cross-sectional, and descriptive. We designed three questionnaires, one for each member of a traditional, el- 
Gonzalo Haro Álvarez, Milagros Bajo Ortega

La comunicación como medio para la cohesión social en las familias tradicionales

ementary family group (father, mother, and child). We then distributed these questionnaires among families in the community of Los Ángeles del Triunfo, in the city of Guasave, Sinaloa, in northwestern Mexico. Our results show that mothers communicate more with their husbands and children, while fathers are more distant from their wife and children. Meanwhile, children communicate more with their mothers. We conclude that communication, trust, and tolerance are indispensable for the cohesion of traditional families.

Keywords: traditional family, social cohesion, communication, needs, family context.

\section{A comunicação como meio para a coesão social nas famílias tradicionais \\ Resumo}

A comunicação é um elemento essencial na conduta e comportamento dos membros de uma família, mas para alcançar a coesão familiar será suficiente? O que explicaria a união nas famílias? O objetivo deste trabalho é contextualizar o ambiente da família tradicional a partir das suas necessidades tangíveis e intangíveis para uma melhor comunicação e unidade familiar baseada nos laços sociais, na confiança, nos valores partilhados e no sentimento de pertença. A metodologia é quantitativa, transversal e descritiva, são elaborados três questionários, um para cada membro (pai, mãe e filho) que constituem a família tradicional da comunidade Los Angeles del Triunfo, Guasave, Sinaloa (México). Nos resultados, ressalta-se que a mãe mantém maior comunicação com o marido e filhos e o pai apresentou menor proximidade com a esposa e filhos e os filhos se comunicam mais com a mãe. Comunicação, confiança e tolerância são imprescindíveis para a coesão das famílias tradicionais.

Palavras chave: família tradicional, coesão social, comunicação, necessidades, contexto familiar.

\section{Introducción}

La familia es reconocida socialmente como un grupo que genera valores de una generación a otra. Es la más antigua de las instituciones humanas; en ella se interactúa, se establecen normas de conducta y se emplea el lenguaje de la comunicación para llegar a acuerdos en común que beneficien a sus integrantes (Benítez, 2017; Morales, 2015; Oliva y Villa, 2014). Su concepto por naturaleza se asocia a la interacción entre sus integrantes, tal como lo definen Oliva y Villa (2014):

[...] es un sistema natural de seres humanos en el cual las personas se encuentran relacionadas por medio de lazos sanguíneos y de afinidad, reunidos en un lugar común delimitado cultural y geográficamente para satisfacer las necesidades básicas, físicas y psicológicas de sus miembros. (p. 14)

No obstante, cobra un papel relevante en aspectos políticos, socioculturales y psicoemocionales que influyen en la sociedad. Mendoza (2020, p. 13) define "familia" como "la unidad fundamental de la sociedad y el medio por naturaleza de crecimiento, apoyo y bienestar, entrelazadas en lo personal y lo social [...] determinando procesos que impactan en la misma sociedad", o, como señala Haro (2019), el papel de la familia es el vínculo para una sociedad cohesionada, es en ella donde los individuos se convierten en agentes de desarrollo o del cambio social. 


\section{Austral Omunicación \\ Volumen 10, número 2 (Diciembre de 2021): 441-459 ISSN (I) 2313-9129. ISSN (E) 2313-9137}

Este grupo ha evolucionado con el paso del tiempo, al igual que las sociedades, al pasar de ser tradicional a moderno (Durkheim, 1967; Gutiérrez et al., 2016). Antiguamente, se hacía referencia a las familias por su tipología, como consanguínea, punalúa, sindiásmica, patriarcal y monógama (Cadenas, 2015; Morales, 2015). Actualmente, se habla de arreglos familiares o diversificación de la familia en su tipo monoparental, homoparental y extensa, entre otros. La más representativa para la sociedad es la familia nuclear, biparental o tradicional, conformada por papá y mamá, además de un número de hijos -como mínimo uno-, que viven juntos (Ariza y De Oliveira, 2007; Benítez, 2017; Esteinou, 2004; Lévi-Strauss, 2010). Este tipo de familia, cuando sufre el divorcio de los padres, el abandono de los hijos, las adicciones, el embarazo precoz, entre otras situaciones, registra deterioro de los lazos familiares (Tapia-Rodríguez, 2021; Valdés et al., 2011).

Castro et al. (2021), Crespo (2011), Santana (2019) y Villavicencio y Villaroel (2017), entre otros, enfatizan la comunicación como esencia en la dinámica familiar para hacer frente a las adversidades; sin ella, se presentarían problemas de conducta entre sus integrantes. En palabras de Gómez (2016), "la comunicación es tan antigua como el hombre y tan importante como él, pues muchos problemas y conflictos se resolverían democráticamente, si este se escuchara con respeto y tolerancia" (p. 5). Es decir, la comunicación es el vehículo para establecer acuerdos e interaccionar en la sociedad. No solo es una necesidad emergente, sino también una forma de relacionarse, de expresar opiniones y de fortalecer el lazo social en individuos que participan en la sociedad (Esteinou, 2004), a través de la familia, amigos, vecinos, grupos u organizaciones, en el que se expresan diferencias y se pueden establecer acuerdos sociales (Durkheim, 1967). También la comunicación puede darse de forma verbal o no verbal entre el que quiere expresar una idea y quien la recibe; a su vez, establece lazos con otras personas creando distintos tipos de conexiones, manifestando inquietudes o necesidades, siendo pruebas de confianza a la espera de que la otra persona capte y contribuya a la situación (Costa et al., 2019).

En este sentido, la comunicación en una familia tradicional se fortalece a través del diálogo, de compartir sus necesidades materiales, como la compra de una casa o la adquisición de otros bienes, y emocionales, como amor, confianza, respeto e identidad (Luengo y Luzón, 2001).

Estas necesidades materiales o emocionales son parte de la cohesión social desde el enfoque no normativo -percepción y contextualización del entorno del sujeto-, en el que Haro y Vázquez (2018) la definen como:

un proceso en el que la organización, participación e integración de los individuos [...] pueden solventar sus necesidades tangibles o intangibles desde el aspecto microsocial, teniendo como pilares los vínculos sociales, confianza, valores compartidos y sentido de pertenencia, mientras estén dispuestos a mantener y renovar dichos pilares. (p. 146) 
Gonzalo Haro Álvarez, Milagros Bajo Ortega

La comunicación como medio para la cohesión social en las familias tradicionales

Es decir, la cohesión social puede ser estudiada a nivel micro, como la familia, para conocer lo que perciben sus integrantes, contextualizar su entorno familiar a partir de sus necesidades materiales y emocionales.

Como se ha señalado, la comunicación es esencial, pero ¿es suficiente para cohesionar a una familia? Daudinot (2012) plantea que la familia es un equipo, en el que se transmiten conocimientos, sentimientos, afecto, confianza y en el que se les da la plena seguridad a sus integrantes de que estarán dispuestos a ayudar al prójimo ante cualquier situación que se les presente, destacando el valor de la solidaridad. Cadenas (2015) menciona que la comunicación permite establecer acuerdos familiares -a partir de su funcionalidad diferenciada- y crear vínculos con los demás miembros de la sociedad, incluyendo a personas desconocidas.

La comunicación no solo se realiza de manera presencial, cara a cara, también se lleva a cabo por medio de llamadas telefónicas o plataformas virtuales (Cuadrado et al., 2015; López, 2015). Siguiendo a Durkheim (1967), aunque las sociedades se modernicen, la solidaridad orgánica como proceso de individuación es una necesidad de cooperación entre individuos de solidarizarse y coordinarse. Este último cobra más sentido en los integrantes de las familias, pues la necesidad de coordinarse es por medio de la comunicación.

Asimismo, Gómez (2008) añade que la convivencia incentiva la identidad y la unión entre padres e hijos adolescentes, generada por un sentimiento compartido, expresada en necesidades de ser escuchados, de compartir experiencias positivas, como logros y metas alcanzadas; incluso de experiencias negativas, como hablar de desempleo, inseguridad, problemas económicos y emocionales, entre otros temas que contextualizan el entorno de los integrantes. Es decir, externar la comunicación mantiene entre los individuos una buena relación, en la que no solo se habla de lo material, sino también de lo sentimental o emocional (Azpiazu et al., 2015).

Desde el punto de vista emocional, Guzmán et al. (2019) afirman que la comunicación influye en el comportamiento de los padres hacia los hijos, pues la familia es la primera escuela. En ella se les inculcan valores a los hijos, afectos, comportamientos y metas en grupos o de manera individual. Es decir, la comunicación es tan importante que un mismo suceso puede generar emociones diferentes en distintas personas (Oliva y Villa, 2014; Suárez y Vélez, 2018).

Es importante señalar el rol que cumplen las emociones en la unión familiar, pues determina que toda persona sin distinción alguna tenga esa característica que es propia de cada uno, esa sensación de afecto hacia algo o alguien, pero cabe destacar que un sentimiento hacia otra persona no solo se da en parejas, sino que esa sensación de cariño y aprecio nace de la comunicación e interacción entre padres e hijos (Bejarano et al., 2021; Gomila, 2005; Márquez-Cervantes y Gaeta-González, 2017). 
Austral Cmunicación

Volumen 10, número 2 (Diciembre de 2021): 441-459 ISSN (I) 2313-9129. ISSN (E) 2313-9137

Es así como la contextualización en el núcleo familiar cobra vital relevancia. Para este estudio, se toma como base la metodología propuesta por Haro y Vázquez (2018) -con énfasis en la comunicación- con la finalidad de contextualizar el entorno de los integrantes de la familia tradicional a partir de sus necesidades tangibles e intangibles para una mejor comunicación y unión familiar basados en el vínculo social, en la confianza, en los valores compartidos y en el sentido de pertenencia (Tabla 1).

Tabla 1. Conceptos, objetivos y contribución de la comunicación en la cohesión de las familias tradicionales

\begin{tabular}{|c|c|c|c|}
\hline Pilares de la CS & Concepto & Objetivo & En qué contribuye \\
\hline Vínculo social & $\begin{array}{l}\text { Compromiso de los } \\
\text { integrantes de la } \\
\text { familia tradicional para } \\
\text { comunicarse de } \\
\text { manera frecuente yasí } \\
\text { llevar a cabo acciones } \\
\text { para una convivencia } \\
\text { sana. }\end{array}$ & $\begin{array}{l}\text { Conocer las } \\
\text { necesidades que } \\
\text { manifiestan las } \\
\text { familias cada vez que } \\
\text { se reúnen, así como } \\
\text { las acciones que llevan } \\
\text { a cabo para lograr una } \\
\text { mejor convivencia y la } \\
\text { importancia de } \\
\text { fomentar la } \\
\text { comunicación para la } \\
\text { solucion de } \\
\text { problemas. }\end{array}$ & $\begin{array}{l}\text { Refuerza la unión entre } \\
\text { sus integrantes a partir } \\
\text { de interactuar y } \\
\text { comunicarse entre sí. } \\
\end{array}$ \\
\hline Confianza & $\begin{array}{l}\text { Expectativas entre los } \\
\text { integrantes de la } \\
\text { familia tradicional } \\
\text { conducidos por la } \\
\text { comunicación y sus } \\
\text { necesidades, con el fin } \\
\text { de resolver sus } \\
\text { problemas } \\
\text { económicos y/o } \\
\text { emocionales. }\end{array}$ & $\begin{array}{l}\text { Conocer el grado de } \\
\text { confianza de la familia } \\
\text { tradicional a partir de } \\
\text { las expectativas de } \\
\text { apoyo entre sus } \\
\text { integrantes en relación } \\
\text { con sus necesidades } \\
\text { materiales o } \\
\text { emocionales. }\end{array}$ & $\begin{array}{l}\text { Incentiva la unión de } \\
\text { sus integrantes a partir } \\
\text { de la comunicación y } \\
\text { valoración que realizan } \\
\text { en la familia, guiados } \\
\text { por las expectativas de } \\
\text { apoyo mutuo. }\end{array}$ \\
\hline Valores compartidos & $\begin{array}{l}\text { Disposición de } \\
\text { solidarizarse a partir de } \\
\text { expresar sus } \\
\text { necesidades, asi como } \\
\text { la tolerancia de } \\
\text { escuchar yel respeto } \\
\text { hacia sus integrantes. }\end{array}$ & $\begin{array}{l}\text { Conocer la intensidad } \\
\text { en valores como } \\
\text { solidaridad, tolerancia } \\
\text { y respeto a partir de } \\
\text { expresar sus } \\
\text { necesidades yen la } \\
\text { acción de sus } \\
\text { integrantes. }\end{array}$ & $\begin{array}{l}\text { Mejora la unión de sus } \\
\text { integrantes a partir de } \\
\text { las metas en común y } \\
\text { de la disposición que } \\
\text { tengan para ser } \\
\text { solidarios ante las } \\
\text { necesidades que les } \\
\text { comuniquen los } \\
\text { demás. }\end{array}$ \\
\hline $\begin{array}{l}\text { Sentido de } \\
\text { pertenencia }\end{array}$ & $\begin{array}{l}\text { Identidad que se } \\
\text { genera a través del } \\
\text { lazo familiar, de } \\
\text { expresar sus } \\
\text { necesidades } \\
\text { emocionales yel } \\
\text { deseo de una mayor } \\
\text { integración. }\end{array}$ & $\begin{array}{l}\text { Conocer las } \\
\text { perspectivas de los } \\
\text { integrantes de la } \\
\text { familia tradicional en } \\
\text { relación con sus } \\
\text { gustos, participación, } \\
\text { integración y los } \\
\text { motivos que los llevan } \\
\text { a generar identidad por } \\
\text { medio de la } \\
\text { comunicación. }\end{array}$ & $\begin{array}{l}\text { Fortalece la unión entre } \\
\text { sus integrantes, } \\
\text { identificados por sus } \\
\text { necesidades, } \\
\text { involucramiento y } \\
\text { compromisos en la } \\
\text { familia. }\end{array}$ \\
\hline
\end{tabular}

Fuente: elaboración propia con base en Haro y Vázquez (2018). 
Gonzalo Haro Álvarez, Milagros Bajo Ortega

La comunicación como medio para la cohesión social en las familias tradicionales

\section{Método}

El estudio es cualitativo y transversal, con la finalidad de contextualizar el entorno de las familias tradicionales de la comunidad los Ángeles del Triunfo, Guasave, Sinaloa (México). Se diseñó un cuestionario semiabierto con preguntas cerradas en la escala de Likert, nominal, dicotómica y ordinal, y preguntas abiertas para conocer más sobre sus necesidades e interacción por medio de la comunicación. El cuestionario contenía cinco apartados: datos generales, vínculos sociales, confianza, valores compartidos y sentido de pertenencia, estos últimos cuatro como los pilares de la cohesión social. Se realizaron tres tipos de cuestionarios para cada uno de los integrantes de las familias tradicionales (papá, mamá e hijos) seleccionadas.

Para llevar a cabo lo anterior, los criterios que se consideraron para la elección de la familia tradicional consistían en que debían vivir juntos papá, mamá e hijos. Dada la complejidad para determinar el número de familias tradicionales a entrevistar, se utilizó el método de muestreo no probabilístico convencional, en el que se logró aplicar diez cuestionarios a familias tradicionales.

La Tabla 2 muestra los pilares de la cohesión social, definición operacional, etiqueta y tipo de escala, con la finalidad de valorar la participación de los miembros de las familias tradicionales a partir de su interacción y comunicación.

\section{Resultados}

En este apartado se presentan los resultados de las entrevistas que se les realizaron a las familias tradicionales, con la finalidad de contextualizar el entorno de sus integrantes a partir de sus necesidades tangibles e intangibles para una mejor comunicación y unión familiar. Primero, se presentan los datos generales de las familias, seguidos de los pilares de cohesión social: vínculo social, confianza, valores compartidos y sentido de pertenencia.

Tabla 2. Estructura operacional

\begin{tabular}{|c|c|c|c|}
\hline $\begin{array}{l}\text { Pilares de la cohesión } \\
\text { social }\end{array}$ & $\begin{array}{l}\text { Definición operacional } \\
\text { (pregunta) }\end{array}$ & Etiqueta & Escala de medición \\
\hline \multirow[b]{2}{*}{ Vinculo social } & $\begin{array}{l}\text { ¿Practica la comunicación } \\
\text { para solucionar los } \\
\text { problemas familiares? }\end{array}$ & $\begin{array}{l}1=\mathrm{Sí} \\
2=\mathrm{No}\end{array}$ & Dicotómica \\
\hline & $\begin{array}{l}\text { ¿Cómo definiría la } \\
\text { relación con su esposo e } \\
\text { hijos? }\end{array}$ & $\begin{array}{l}\text { 1= Excelente } \\
2=\text { Buena } \\
3=\text { Regular } \\
4=\text { Mala }\end{array}$ & Ordinal \\
\hline
\end{tabular}


Austral Comunicación

Volumen 10, número 2 (Diciembre de 2021): 441-459

ISSN (I) 2313-9129. ISSN (E) 2313-9137

\begin{tabular}{|c|c|c|c|}
\hline $\begin{array}{l}\text { Pilares de la cohesión } \\
\text { social }\end{array}$ & $\begin{array}{c}\text { Definición operacional } \\
\text { (pregunta) }\end{array}$ & Etiqueta & Escala de medición \\
\hline \multirow{8}{*}{ Confianza } & $\begin{array}{l}\text { ¿Cómo mejora la relación } \\
\text { con su familia? }\end{array}$ & $\begin{array}{l}\text { 1= Hablando con } \\
\text { honestidad y sinceridad } \\
2=\text { Respetando el tiempo } \\
\text { del otro } \\
3=\text { Tolerando gustos } \\
\text { diferentes } \\
\text { 4= Pasando tiempo en } \\
\text { familia } \\
5=\text { Todos }\end{array}$ & Nominal \\
\hline & $\begin{array}{l}\text { ¿Qué tanto considera que } \\
\text { su familia lo ha apoyado } \\
\text { en sus necesidades? }\end{array}$ & $\begin{array}{l}\text { 1= Muypoco } \\
2=\text { Poco } \\
3=\text { Medianamente } \\
4=\text { Suficiente } \\
5=\text { Mucho }\end{array}$ & Likert \\
\hline & $\begin{array}{l}\text { ¿Qué tan importante ha } \\
\text { sido la ayuda que ha } \\
\text { recibido? }\end{array}$ & $\begin{array}{l}1=\text { Muy poco importante } \\
2=\text { Poco importante } \\
3=\text { Ni muy poco ni muy } \\
\text { importante } \\
4=\text { Importante } \\
5=\text { Muy importante }\end{array}$ & Likert \\
\hline & $\begin{array}{l}\text { ¿Apoya emocionalm ente } \\
\text { a su esposo e hijos? }\end{array}$ & $\begin{array}{l}1=S i ́ \\
2=\text { No }\end{array}$ & Dicotómica \\
\hline & $\begin{array}{l}\text { ¿Qué tanta comunicación } \\
\text { mantiene con su } \\
\text { esposo(a)? }\end{array}$ & $\begin{array}{l}\text { 1= Me comunico } \\
\text { frecuentemente } \\
2=\text { Apenas me com unico } \\
3=\text { No me comunico }\end{array}$ & Ordinal \\
\hline & $\begin{array}{l}\text { ¿Qué tanta com unicación } \\
\text { mantiene con sus hijos? }\end{array}$ & $\begin{array}{l}1=\text { Me comunico } \\
\text { frecuentemente } \\
2=\text { Apenas me com unico } \\
3=\text { No me comunico }\end{array}$ & Ordinal \\
\hline & $\begin{array}{l}\text { ¿Con qué familiar suele } \\
\text { tener más comunicación? }\end{array}$ & $\begin{array}{l}1=\text { Esposo } \\
2=\text { Esposa } \\
3=H i j o\end{array}$ & Nominal \\
\hline & $\begin{array}{l}\text { ¿Qué valores identifica en } \\
\text { su familia? }\end{array}$ & $\begin{array}{l}1=\text { Empatía } \\
2=\text { Amor } \\
3=\text { Bondad } \\
4=\text { Humildad } \\
5=\text { Responsabilidad } \\
6=\text { Solidaridad } \\
7=\text { Ninguno }\end{array}$ & Nominal \\
\hline \multirow[b]{2}{*}{ Valores compartidos } & $\begin{array}{l}\text { ¿Está usted de acuerdo } \\
\text { con la siguiente } \\
\text { afirmación: "participar en } \\
\text { actividades que } \\
\text { beneficien a los } \\
\text { integrantes de mi familia } \\
\text { es muy importante"? }\end{array}$ & $\begin{array}{l}1=\text { Totalmente de acuerdo } \\
2=\text { Totalmente en } \\
\text { desacuerdo }\end{array}$ & Dicotómica \\
\hline & $\begin{array}{l}\text { ¿Qué tan tolerante se } \\
\text { considera con su } \\
\text { esposo? }\end{array}$ & $\begin{array}{l}\text { 1= Muypoco } \\
2=\text { Poco } \\
3=\text { Medianamente } \\
\text { 4= Suficiente } \\
5=\text { Mucho }\end{array}$ & Ordinal \\
\hline
\end{tabular}


Gonzalo Haro Álvarez, Milagros Bajo Ortega

La comunicación como medio para la cohesión social en las familias tradicionales

\begin{tabular}{|c|c|c|c|}
\hline $\begin{array}{l}\text { Pilares de la cohesión } \\
\text { social }\end{array}$ & $\begin{array}{l}\text { Definición operacional } \\
\text { (pregunta) }\end{array}$ & Etiqueta & Escala de medición \\
\hline Valores compartidos & $\begin{array}{l}\text { ¿Qué tan tolerante se } \\
\text { considera con sus hijos? }\end{array}$ & $\begin{array}{l}\text { 1= Muy poco } \\
2=\text { Poco } \\
3=\text { Medianamente } \\
\text { 4= Suficiente } \\
5=\text { Mucho }\end{array}$ & Ordinal \\
\hline \multirow{5}{*}{ Sentido de pertenencia } & $\begin{array}{l}\text { ¿Qué tanto se identifica } \\
\text { con su esposo? }\end{array}$ & $\begin{array}{l}\text { 1= Muy poco identificado } \\
\text { 2= Poco identificado } \\
3=\text { Ni muy poco ni muy } \\
\text { identificado } \\
\text { 4= Identificado } \\
5=\text { Muy identificado }\end{array}$ & Likert \\
\hline & $\begin{array}{l}\text { ¿Qué tanto se identifica } \\
\text { con sus hijos? }\end{array}$ & $\begin{array}{l}\text { 1= Muy poco identificado } \\
2=\text { Poco identificado } \\
3=\text { Ni muy poco ni muy } \\
\text { identificado } \\
\text { 4= Identificado } \\
5=\text { Muy identificado }\end{array}$ & Likert \\
\hline & $\begin{array}{l}\text { ¿Desearía una mayor } \\
\text { integración con su } \\
\text { familia? }\end{array}$ & $\begin{array}{l}1=S i ́ \\
2=N o\end{array}$ & Dicotómica \\
\hline & $\begin{array}{l}\text { ¿Cómo se entera de las } \\
\text { necesidades de cada } \\
\text { miembro de su familia? }\end{array}$ & $\begin{array}{l}1=\text { Yo les pregunto } \\
2=\text { Ellos me lo dicen } \\
3=\text { Nunca me entero }\end{array}$ & Nominal \\
\hline & $\begin{array}{l}\text { ¿Le gustaria interactuar } \\
\text { más tiempo con su } \\
\text { familia? }\end{array}$ & $\begin{array}{l}1=\mathrm{Sí} \\
2=\mathrm{No}\end{array}$ & Dicotómica \\
\hline
\end{tabular}

Fuente: elaboración propia con base en el instrumento aplicado.

\section{Datos generales}

La Tabla 3 muestra las características generales de las familias tradicionales entrevistadas en relación con el porcentaje de nivel de estudios y promedio de edad. Al respecto, el 30\% de los hijos estudian la secundaria; y el 60\%, la licenciatura. En el caso del papá, hay un comportamiento similar en el nivel de estudios, resaltando asimismo que el $30 \%$ de las madres cuenta con estudios de licenciatura. Con respecto a la edad, se puede decir que son relativamente jóvenes, obteniendo edades medias de 45,7 años para la mamá y 47,3 años para el papá. La desviación estándar de los padres indica que se registraron rangos más amplios de edades en la mamá que en el papá. La edad de los hijos está entre la adolescencia y la juventud.

\section{Vínculos sociales}

El primer pilar tiene por objetivo conocer las necesidades de las que hablan las familias cada vez que se reúnen, así como las acciones que llevan a cabo para una mejor convivencia y la importancia de fomentar la comunicación para la solución de problemas. 
Austral Comunicación

Volumen 10, número 2 (Diciembre de 2021): 441-459

ISSN (I) 2313-9129. ISSN (E) 2313-9137

Tabla 3. Características generales de las familias tradicionales (\%)

\begin{tabular}{lccl}
\hline \multicolumn{1}{c}{ Estudio } & Mamá & Papá & Hijo \\
\hline Primaria & 30 & 40 & \\
\hline Secundaria & 20 & 10 & 30 \\
\hline Preparatoria & 20 & 30 & 10 \\
\hline Licenciatura & 30 & 20 & 60 \\
\hline Edad promedio & 45,7 & 47,3 & 18 \\
\hline Desviación estándar & 6,18 & 4,83 & 2,91 \\
\hline
\end{tabular}

Fuente: elaboración propia con base en el instrumento aplicado.

Está estructurado en cinco reactivos: tres en formato de pregunta abierta, con la finalidad de contextualizar el entorno de los integrantes, y dos preguntas cerradas en escala dicotómica (1) y ordinal (1). En este sentido, la primera pregunta que se planteó fue: ¿sobre qué necesidades hablan en su familia cada vez que se reúnen? Las principales respuestas que se presentaron con mayor frecuencia fueron "económica" y "salud"; la segunda pregunta fue: ¿qué acciones lleva a cabo en su familia para lograr una convivencia feliz? Las respuestas que se mencionaron fueron "fomentar la comunicación para estar juntos" y realizar actividades en familia como "salir a comer", "pasear y realizar compras". La tercera pregunta fue: ¿por qué es tan importante que haya comunicación en su familia? Las respuestas giraron en torno a "es primordial para apoyarnos", "para que no haya malas interpretaciones", "para que haya buena convivencia", "una mejor relación, armonía", "para entendernos mejor", "para mantenernos unidos e integrados".

Posteriormente, se realizaron dos preguntas con opción múltiple, en las cuales el $100 \%$ de las madres de las familias encuestadas de la comunidad respondieron que recurren a la comunicación como medio para la solución de sus conflictos; lo mismo respondieron el $90 \%$ de los papás y el $80 \%$ de los hijos encuestados. Además, las mamás presentaron mayor participación en impulsar a los demás integrantes de la familia a comunicar sus problemas o inquietudes para mantenerse unidos, así como pasar tiempo juntos y realizar actividades que le agrade a su familia con el propósito de convivir, comunicarse, mantenerse integrados y felices.

En cuanto a la relación de pareja, el $60 \%$ de las esposas mencionó que tiene una excelente relación con su esposo, y el $30 \%$ consideran que es buena. En cuanto al esposo, el $60 \%$ mencionó que la relación con su esposa es buena; y el $40 \%$, excelente. Con respecto a la relación de los padres con sus hijos, el $60 \%$ contestó que es excelente y el 
Gonzalo Haro Álvarez, Milagros Bajo Ortega

La comunicación como medio para la cohesión social en las familias tradicionales

$30 \%$ que es buena. En el caso de los hijos, mencionaron que la relación con sus padres es buena ( $40 \%)$ o excelente (60\%). Por último, todos los encuestados manifestaron que es importante fomentar la comunicación para conocer las necesidades de cada uno y buscar soluciones en conjunto en caso de conflicto (Tabla 4).

Tabla 4. Vínculos sociales

\begin{tabular}{lccc}
\hline \multirow{2}{*}{ Indicadores } & Mamá (\%) & Papá (\%) & Hijo (\%) \\
\hline \multirow{2}{*}{ Sí } & \multicolumn{2}{c}{ ¿Practica la comunicación para solucionar los problemas familiares? } \\
\cline { 2 - 4 } & 100 & 90 & 80 \\
\hline \multirow{2}{*}{ Excelente } & \multicolumn{2}{c}{ ¿Cómo definiría la relación con su esposo e hijos? } \\
\cline { 2 - 3 } & 60 & 40 & 60 \\
\hline & 30 & 60 & 40 \\
\hline
\end{tabular}

Fuente: elaboración propia con base en el instrumento aplicado.

\section{Confianza}

El segundo pilar tiene como objetivo conocer el grado de confianza de la familia tradicional a partir de sus expectativas de apoyo entre sus integrantes en relación con sus necesidades materiales o emocionales.

Está estructurado en diez reactivos: tres en formato de pregunta abierta, con la finalidad de hacer notar el entorno de la mamá, papá e hijos, y siete preguntas cerradas en escala nominal (2), Likert (2), ordinal (2) y dicotómica (1). En este sentido, se les preguntó acerca de cómo mejorarían la relación en su familia, a lo cual el $80 \%$ de las mamás, el $90 \%$ de los papás y el $60 \%$ de los hijos mencionaron que hablando con honestidad y sinceridad, tolerando gustos diferentes y pasando tiempo en familia. El 90\% de los papás coinciden con las respuestas de sus esposas, lo que indica que la mayoría de los padres de familia (mamá y papá) piensan de la misma manera cuando expresan que todas las opciones son fundamentales para mejorar la relación entre ellos. En el caso de los hijos, el 10\% respondió que se logra hablando con honestidad y sinceridad, el $20 \%$ lo hace tolerando gustos diferentes; otro $10 \%$, pasando tiempo en familia y el $60 \%$ coincide con las respuestas de sus padres.

Además, es importante conocer qué tanto se han apoyado en sus necesidades. Las respuestas predominantes variaron entre dos opciones: suficiente o mucho. El $40 \%$ de las mamás indicó que ha sido suficiente el apoyo que ha recibido por parte de su familia, mientras que el 60\% mencionó que es mucho el apoyo. El 50\% de los papás señala suficiente; y el 30\%, que ha sido mucho. En el caso de los hijos, el 70\% manifestó que ha sido mucho lo que su familia los ha apoyado en sus necesidades. 


\section{Austral Omunicación \\ Volumen 10, número 2 (Diciembre de 2021): 441-459 ISSN (I) 2313-9129. ISSN (E) 2313-9137}

Con la finalidad de contextualizar su entorno familiar, se plantearon dos preguntas abiertas por cada integrante. En el caso de la esposa se le preguntó: ¿qué tipo de ayuda recibe por parte de su esposo? y ¿qué tipo de ayuda recibe por parte de su hijo? Para la primera pregunta, las respuestas más comunes fueron "económicas y emocionales", y para la segunda, "educación y emocional". Las mismas preguntas se plantearon los papás con relación a su esposa e hijos, respondiendo a la primera pregunta "me escucha y me aconseja", y para la segunda, "emocional, me da ánimos". De la misma manera, se plantearon preguntas a los hijos: ¿qué tipo de ayuda recibe por parte de su papá? y ¿qué tipo de ayuda recibe por parte de su mamá? Para la primera pregunta, respondieron "necesidades básicas, me da mis estudios", y para la segunda, "me escucha cuando la necesito, me aconseja”.

Asimismo, en relación con las expectativas de apoyos que el individuo tiene respecto a sus familiares, se les preguntó: ¿qué tan importante ha sido la ayuda que ha recibido? El $80 \%$ de las mamás consideran que la ayuda que han recibido por parte de su familia es muy importante. En cuanto a los papás, tuvieron un cierto balance en sus respuestas debido a que el 50\% indicó importante; y el otro 50\%, muy importante. Y de los hijos, el 60\% dijo muy importante; y el 30\%, importante.

En cuanto a las acciones que necesita la familia tradicional para que confíen más en ellos, las respuestas de la mamá, papá e hijo giraron en torno a "fomentar la comunicación, apoyo y compañía”. Además, mencionaron que para fortalecer el sentimiento de confianza se requiere "comunicación constante, fomentar las pláticas, que platiquen sus inquietudes, mayor diálogo, respeto, comunicación, amor, atención, honestidad, actitud, convivencia y mayor comprensión".

Posteriormente, desde el aspecto de apoyo emocional, el 100\% de las mamás, el $90 \%$ de los papás y el $100 \%$ de los hijos mencionaron apoyarse emocionalmente. Este tipo de resultados obedece a la comunicación que existe en la familia. Por último, las mamás indicaron que tienen la misma comunicación con su esposo e hijos (80\%), los papás con su esposa (90\%), y los hijos con su mamá (Tabla 5).

\section{Valores compartidos}

El tercer pilar tiene por objetivo conocer la intensidad en valores como solidaridad, tolerancia y respeto a partir de expresar sus necesidades y en la acción de sus integrantes.

Está estructurado en seis reactivos: dos en formato de pregunta abierta y cuatro preguntas cerradas en escala nominal (1), dicotómica (1) y ordinal (2). En este sentido, la primera pregunta que se planteó fue: ¿qué valores identifica en su familia? El 50\% de las mamás, el $70 \%$ de los papás y el $50 \%$ de los hijos respondieron que el valor que identifican en su familia es el amor. 
Gonzalo Haro Álvarez, Milagros Bajo Ortega

La comunicación como medio para la cohesión social en las familias tradicionales

Tabla 5. Indicadores de confianza en la familia tradicional

\begin{tabular}{|c|c|c|c|}
\hline Indicadores & Mamá (\%) & Papá (\%) & Hijo (\%) \\
\hline & \multicolumn{3}{|c|}{ ¿Cómo mejora la relación con su familia? } \\
\hline $\begin{array}{l}\text { Hablando con honestidad } \\
\text { y sinceridad }\end{array}$ & 10 & & 10 \\
\hline Tolerando gustos diferentes & & & 20 \\
\hline Pasando tiempo en familia & 10 & 10 & 10 \\
\hline \multirow[t]{2}{*}{ Todos } & 80 & 90 & 60 \\
\hline & \multicolumn{3}{|c|}{ ¿Qué tanto considera que su familia lo ha apoyado en sus necesidades? } \\
\hline Suficiente & 40 & 50 & 20 \\
\hline \multirow[t]{2}{*}{ Mucho } & 60 & 30 & 70 \\
\hline & \multicolumn{3}{|c|}{ ¿Qué tipo de ayuda recibe de cada uno? } \\
\hline Importante & 20 & 50 & 30 \\
\hline \multirow[t]{2}{*}{ Muy importante } & 80 & 50 & 60 \\
\hline & \multicolumn{3}{|c|}{ ¿Apoya emocionalmente a su esposo/a e hijos? } \\
\hline \multirow[t]{2}{*}{ Sí } & 100 & 90 & \\
\hline & \multicolumn{3}{|c|}{ ¿Con qué familiar suele tener más comunicación? } \\
\hline Esposa & & 90 & 80 \\
\hline Esposo & 80 & & 20 \\
\hline Hijo & 80 & 20 & \\
\hline
\end{tabular}

Fuente: elaboración propia con base en el instrumento aplicado.

Además se les preguntó: ¿qué tipo de ayuda está dispuesto a ofrecerle a su familia? La respuesta que mayor predominó en mamá, papá e hijo fue "la que ellos necesiten y todo tipo de ayuda”. Además, están de acuerdo en participar en actividades que beneficien a los demás integrantes de su familia, ya que consideran que es muy importante para una mayor unión. Asimismo, se les preguntó acerca de las actividades que contribuirían a una mejor integración familiar. Las respuestas en común fueron "salir a pasear juntos", "convivencia diaria", "cocinar" y "practicar deporte". Con respecto a la tolerancia, los esposos mencionaron suficiente o mucha, y de los hijos hacia sus padres, el 70\% mencionó que es mucha (Tabla 6).

\section{Sentido de pertenencia}

El cuarto pilar tiene por objetivo conocer las perspectivas de los integrantes de la familia tradicional en relación con sus gustos, participación, integración y los motivos que los llevan a generar identidad por medio de la comunicación. Está estructurado en trece reactivos: ocho en formato de pregunta abierta, con la finalidad de contextualizar 
Austral Comunicación

Volumen 10, número 2 (Diciembre de 2021): 441-459

ISSN (I) 2313-9129. ISSN (E) 2313-9137

Tabla 6. Valores compartidos

\begin{tabular}{|c|c|c|c|}
\hline Indicadores & Mamá (\%) & Papá (\%) & Hijo (\%) \\
\hline & \multicolumn{3}{|c|}{ ¿Qué valores identifica en su familia? } \\
\hline Empatía & 20 & & 20 \\
\hline Amor & 50 & 70 & 50 \\
\hline Responsabilidad & 10 & & 10 \\
\hline \multirow[t]{2}{*}{ Solidaridad } & 20 & 30 & 20 \\
\hline & \multicolumn{3}{|c|}{$\begin{array}{l}\text { ¿Está usted de acuerdo con la sıgulente afırmación?: } \\
\text { "Participar en actividades que beneficien a los integrantes } \\
\text { de mi familia es muy importante" }\end{array}$} \\
\hline Sí & 100 & 100 & 100 \\
\hline \multirow{11}{*}{$\begin{array}{l}\text { ¿Qué tan tolerante } \\
\text { se considera } \\
\text { con...? }\end{array}$} & Mamá & Tolerancia esposo & Tolerancia hijos \\
\hline & Medianamente & 20 & 10 \\
\hline & Suficiente & 50 & 40 \\
\hline & Mucho & 30 & 50 \\
\hline & Papá & Tolerancia esposa & Tolerancia hijos \\
\hline & Suficiente & 40 & 50 \\
\hline & Mucho & 40 & 50 \\
\hline & Hijo & Tolerancia papá & Tolerancia mamá \\
\hline & Medianamente & 40 & 10 \\
\hline & Suficiente & 60 & 20 \\
\hline & Mucho & & 70 \\
\hline
\end{tabular}

Fuente: elaboración propia con base en el instrumento aplicado.

el entorno de la mamá, papá e hijos, y cinco preguntas cerradas en escala de Likert (2), nominal (1) y dicotómica (2).

En este sentido, las familias tradicionales mencionaron que se sienten identificadas o muy identificadas con ellos. Además, todos los padres desean una mayor integración con su familia, ya que expresaron: "Se está desintegrando", "me preocupa mantenerla unida", "para que no se pierda la costumbre de comunicarnos", "para la distracción", "porque quiero lo mejor para ellos", "para una convivencia feliz", "para estar bien", "para pasar más tiempo con mi hijo", mientras que el 90\% de los hijos indicaron que para 
Gonzalo Haro Álvarez, Milagros Bajo Ortega

La comunicación como medio para la cohesión social en las familias tradicionales

ellos también es importante mantener siempre la unión "para fortalecer los lazos familiares", "no perder la confianza que se tienen", así como "para entenderse mejor".

Posteriormente se les preguntó: ¿qué representa para ti tu familia? Las respuestas que se presentaron con mayor frecuencia fueron "mi familia representa todo", "mi familia representa cariño y respeto", "es mi refugio, felicidad, mi vida". Otra pregunta fue: ¿cómo se entera de las necesidades de cada miembro de su familia? El 70\% de las mamás, el $50 \%$ de los papás y el $70 \%$ de los hijos dijeron que se enteran porque les preguntan, mientras que también existe otro $50 \%$ de los papás que indicaron "ellos me lo dicen".

Asimismo, el 100\% de las mamás y papás encuestados mencionaron que les gustaría interactuar más tiempo con su familia, como también el 90\% de los hijos. Los comentarios más frecuentes de los entrevistados fueron: "Falta mejorar la comunicación", "nos falta convivencia para conocernos más", "para que haya mayor unión", "para mejorar la integración", "para estar enterada de todo, a veces no tenemos la suficiente comunicación", "para unir a mi familia, no quiero alejarlos de mi vida”.

Posteriormente, se les plantearon tres preguntas abiertas acerca de gustos, molestias y propuestas para mejorar la relación en su familia. En el caso de los gustos, mencionaron: "Me gusta que nos apoyamos los unos a los otros", "están conmigo y me ayudan en lo que necesito", "el que hagan todo por mantenernos unidos" y "me gusta la convivencia sana". En el caso de lo que les molesta: "Que no me den mi espacio personal", "que no coincidamos en nada", "actuar sin pensar". Y en el caso de las propuestas, mencionaron "fomentar la comunicación", "pasar tiempo juntos", "hablar siempre con la verdad", "respetarnos los unos a los otros".

Por último, se indagó acerca de qué tan identificados se sienten entre ellos. El 70\% de las esposas se sienten identificadas o muy identificadas con su esposo, y el $100 \%$ se identifica con sus hijos. En el caso de los esposos, es el mismo porcentaje de identificación con las esposas, y el $80 \%$ se siente identificado o muy identificado con sus hijos. En el caso de los hijos, todos los entrevistados mencionaron que se sienten identificados o muy identificados con su papá; y el $80 \%$, con su mamá (Tabla 7 , ver página siguiente).

\section{Discusión de resultados}

El objetivo de la investigación fue contextualizar el entorno de las familias tradicionales de la comunidad Los Ángeles del Triunfo, Guasave, Sinaloa (México) a partir de sus necesidades tangibles e intangibles. De acuerdo con Haro y Vázquez (2018), la cohesión social puede ser estudiada a nivel microsocial -como la familia- a través de sus cuatro pilares: vínculos sociales, confianza, valores compartidos y sentido de pertenencia. Su riqueza teórica se sustenta en Durkheim (1967), que consideraba que la solidaridad está presente en el ser humano guiado por valores, emociones e ideas, 
Austral Comunicación

Tabla 7. Sentido de pertenencia

\begin{tabular}{|c|c|c|c|}
\hline Indicadores & Mamá (\%) & Papá (\%) & Hijo (\%) \\
\hline & \multicolumn{3}{|c|}{ ¿Desearía una mayor integración con su familia? } \\
\hline \multirow[t]{2}{*}{ Sí } & 100 & 100 & 90 \\
\hline & \multicolumn{3}{|c|}{ ¿Cómo se entera de sus necesidades? } \\
\hline Yo les pregunto & 70 & 50 & 70 \\
\hline Ellos me lo dicen & 20 & 50 & 10 \\
\hline \multirow[t]{2}{*}{ Nunca me entero } & 10 & & 20 \\
\hline & \multicolumn{3}{|c|}{ ¿Le gustaría interactuar más tiempo con su familia? } \\
\hline Sí & 100 & 100 & 90 \\
\hline \multirow{10}{*}{$\begin{array}{l}\text { ¿Qué tanto se } \\
\text { identifica con...? }\end{array}$} & Mamá & Esposo & Hijos \\
\hline & Identificado & 30 & 50 \\
\hline & Muy identificado & 40 & 50 \\
\hline & Papá & Esposa & Hijos \\
\hline & Identificado & 40 & 50 \\
\hline & Muy identificado & 30 & 30 \\
\hline & Hijo & Papá & Mamá \\
\hline & $\begin{array}{l}\mathrm{Ni} \text { muy poco ni muy } \\
\text { identificado }\end{array}$ & & 20 \\
\hline & Identificado & 50 & 20 \\
\hline & Muy identificado & 50 & 60 \\
\hline
\end{tabular}

y aunque las sociedades pasen de ser tradicionales a modernas, la cohesión se puede dar por acuerdos sociales, en los que el individuo participa e interactúa. Asimismo, autores como Crespo (2011) y Villavicencio y Villaroel (2017), entre otros, señalan que la comunicación es la esencia en la dinámica familiar para hacerles frente a las adversidades; sin ella, sus integrantes manifestarían problemas de conducta. En este sentido, los resultados del presente estudio demuestran que la comunicación es el vehículo para fortalecer los lazos sociales entre los integrantes de la familia tradicional y la confianza a través del apoyo mutuo, de escuchar y ser escuchado (Costas et al., 2019), poder expresar una idea y manifestar sus inquietudes o necesidades de tipo económico y/o emocional (Gómez, 2008), de manera tal que el valor del amor (Luengo y Luzón, 2001) 
Gonzalo Haro Álvarez, Milagros Bajo Ortega

La comunicación como medio para la cohesión social en las familias tradicionales

y la solidaridad contribuyan a dar respuesta a situaciones que podrían deteriorar las relaciones o desintegrar a la familia (Daudinot, 2012), así como las diferentes formas de convivencia entre esposo y esposa, padres e hijos, generan emociones diferentes (Oliva y Villa, 2014; Suárez y Vélez, 2018) e incentivan la identidad familiar (Gómez, 2008; Luengo y Luzón, 2001).

\section{Conclusión}

La comunicación concebida como el vehículo para relacionarse y expresar opiniones es vital para el fortalecimiento de los lazos sociales y la relación esposo-esposa y padres e hijos. Con respecto a los pilares de la cohesión social, se puede señalar que en el primero (vínculos sociales), la comunicación frecuente en las familias contribuye a fomentar una mejor convivencia, entendimiento y conocimiento de sus necesidades materiales y/o emocionales. La principal necesidad en las familias fue de tipo económico, y acciones como salir a pasear o ir de compras genera mayor integración. La comunicación es muy importante para conocer las ideas y opiniones de los integrantes, lo que genera mayor relación, armonía y unión.

En el segundo pilar (confianza), el apoyo que reciben los integrantes es vital para alimentar la confianza expresando sus necesidades emocionales, ser escuchados, recibir consejos y ánimos. Realizar actividades juntos, como salir de paseo o de compras y cocinar, une a las familias. La comunicación fortalece la confianza en la esposa y el esposo y su relación con los hijos, lo que propicia un ambiente de felicidad y de emociones positivas. En el tercer pilar (valores compartidos), el amor es el valor más importante para la mamá, papá e hijos. La solidaridad en las familias es consecuencia de expresar sus necesidades, tener tolerancia para escuchar y ser escuchados y transmitir respeto a sus integrantes. Por último, en el cuarto pilar (sentido de pertenencia), la familia representa el espacio para socializar e interactuar, compartir experiencias y necesidades, es el refugio para los hijos, el respeto y la felicidad de sus integrantes, la confianza para expresar sus emociones, el apoyo mutuo para superar adversidades y mayor unión. La identidad se genera por todas las actividades y acciones que realizan en conjunto.

Desde la familia tradicional, las mamás (esposas) son las que más practican la comunicación como medio para la solución de conflictos, ya que impulsan en los demás integrantes la comunicación de sus problemas o inquietudes para mantenerlos unidos. Escuchan y aconsejan al esposo e hijos; se preocupan por mantener unida a su familia, incluso prefieren realizar actividades que le agrade a su familia con el propósito de convivir, mantenerse integrados y felices. El papá (esposo) es el que provee el dinero para el hogar y estudios de los hijos; se preocupa de que tengan lo necesario. Los hijos presentaron muy buena relación con sus padres; independientemente de si están en la 


\section{Austral@municación \\ Volumen 10, número 2 (Diciembre de 2021): 441-459 ISSN (I) 2313-9129. ISSN (E) 2313-9137}

adolescencia o si son jóvenes; son más tolerantes con las mamás y consideran que los padres deberían ser más comprensivos con ellos.

Se concluye que la comunicación es el medio para lograr familias tradicionales más cohesionadas, mejores relaciones entre esposa y esposo y padres e hijos. A su vez, contextualizar el entorno en el que participan sus integrantes contribuye a tener la misma información. El diálogo y la comprensión fortalecen la capacidad de escuchar y ser escuchado. Los valores definen la conducta del individuo. Las formas de convivencia unen a las familias y, por ende, construyen una sociedad más cohesionada.

\section{Referencias}

Ariza, M. y De Oliveira, O. (2007). Familias, pobreza y desigualdad social en Latinoamérica: una mirada comparativa. Estudios Demográficos y Urbanos, 22(1), 9-42. https://doi.org/10.24201/edu.v22i1.1292.

Azpiazu, L., Esnaola, I. y Sarasa, M. (2015). Capacidad predictiva del apoyo social en la inteligencia emocional de adolescentes. European Journal of Education and Psychology, 8(1), 23-29. https://doi.org/10.1016/j.ejeps.2015.10.003.

Bejarano, A., Alba-Beltrán, L. y Pineda, N. (2021). Pautas de crianza en el sector rural. Revista Estudios Psicológicos, 1(2), 66-73. https://doi.org/10.35622/j. rep.2021.02.004.

Benítez, M. E. (2017). La familia: Desde lo tradicional a lo discutible. Revista Novedades en Población, 13(26), 58-68. http://scielo.sld.cu/scielo. php?script=sci_arttext\&pid=S1817-40782017000200005.

Cadenas, H. (2015). La familia como sistema social: Conyugalidad y parentalidad. MAD, (33), 29-41. https://doi.org/10.5354/0718-0527.2015.37322.

Castro, R., Vargas, E. y García, J. U. (2021). El autoconcepto del adolescente, su relación con la comunicación familiar y la violencia escolar. Dilemas Contemporáneos: Educación, Política y Valores, 8(1), 1-14.https://doi.org/10.46377/dilemas.v8i.2570.

Costa, G. M, Teles, I. D., Bernardo, J. A. y Alves, A. G. (2019). Comunicación no verbal en el cuidado de usuarios hipertensos en la Estrategia de Salud de la Familia. Revista Familia, Ciclos de Vida e Saúde no Contexto Social, 7(3), 284-295. https:// www.redalyc.org/jatsRepo/4979/497960141002/497960141002_1.pdf.

Crespo, J. M. (2011). Bases para construir una comunicación positiva en la familia. Revista de Investigación en Educación, 9(2), 91-98. http://revistas.webs.uvigo.es/ index.php/reined/article/view/1891/1802.

Cuadrado, I., Martín-Mora, G. y Fernández, I. (2015). La expresión de las emociones en la Comunicación Virtual: El Ciberhabla. ICONO 14. Revista de Comunicación $y$ Tecnologías Emergentes, 13(1), 180-207. https://doi.org/10.7195/ri14.v13i1.716. 
Gonzalo Haro Álvarez, Milagros Bajo Ortega

La comunicación como medio para la cohesión social en las familias tradicionales

Daudinot, J. (2012). La comunicación intrafamiliar: Una necesidad en la formación inicial del maestro primario. EduSol, 12(40), 42-52. https://www.redalyc.org/ articulo.oa? id=475748679005.

Durkheim, E. (1967). De la división del trabajo social. Schapire.

Esteinou, R. (2004). El surgimiento de la familia nuclear en México. Estudios de Historia Novohispana, (31), 99-136. https://doi.org/10.22201/iih.24486922e.2004.031.3613.

Gómez, E. (2008). Adolescencia y familia: revisión de la relación y la comunicación como factores de riesgo o protección. Revista Internacional de Psicología y Educación, 10(2), 105-102. https://www.redalyc.org/pdf/802/80212387006.pdf.

Gómez, E. (2016). La comunicación. Salus, 20(3), 5-6. http://ve.scielo.org/pdf/s/v20n3/ art02.pdf.

Gomila, M. A. (2005). Las relaciones intergeneracionales en el marco de la familia contemporánea: cambios y continuidades en transición hacia una nueva concepción de la familia. Historia Contemporánea, (31), 505-542. https://ojs.ehu. eus/index.php/HC/article/view/4245.

Gutiérrez, R., Díaz, K. Y. y Román, R. P. (2016). El concepto de familia en México: una revisión desde la mirada antropológica y demográfica. CIENCIA Ergo-Sum, 23(3), 219-228. https://cienciaergosum.uaemex.mx/article/view/7364.

Guzmán K., Bastidas, B. y Mendoza, M. (2019). Estudio del rol de los padres de familia en la vida emocional de los hijos. Apuntes Universitarios. Revista de Investigación, 9(2), 61-72. https://doi.org/10.17162/au.v9i2.360.

Haro, G. (2019). Estimación de la cohesión social no normativa en el municipio de Macuspana, Tabasco. Tla-melaua. Revista de Ciencias Sociales, 13(47), 196-219. http://www.scielo.org.mx/pdf/tla/v13n47/2594-0716-tla-13-47-196.pdf.

Haro, G. y Vázquez, J. D. (2018). La cohesión social desde una perspectiva no normativa alternativa de un diseño instrumental. Tla-melaua. Revista de Ciencias Sociales, 11(43), 132-154. https://doi.org/10.32399/rtla.11.43.373.

Lévi-Strauss, C. (2010). La familia. En Velasco, H. M. (Comp.), Lecturas de antropología social y cultural. La Cultura y las Culturas (pp. 222-195). UNED.

López, J. (2015). La cohesión social desde las redes sociales: El caso de la Calle San Francisco. Universidad de Alicante.

Luengo, J. J. y Luzón, A. (2001). El proceso de transformación de la familia tradicional y sus implicaciones educativas. Investigación en la Escuela, 44, 55-68. https:// revistascientificas.us.es/index.php/IE/article/view/7660.

Márquez-Cervantes, M. C.y Gaeta-González, M.L.(2017). Desarrollo de competencias emocionales en pre-adolescentes: el papel de padres y docentes. Revista Electrónica Interuniversitaria de Formación del Profesorado, 20(2), 221-235. https://revistas. um.es/reifop/article/view/232941. 
Austral Comunicación

Volumen 10, número 2 (Diciembre de 2021): 441-459 ISSN (I) 2313-9129. ISSN (E) 2313-9137

Mendoza, L. A. (2020). Los arreglos familiares en Tamaulipas, México. Revista Latinoamericana de Estudios de Familia, 12(1), 11-31. https://revistasojs.ucaldas. edu.co/index.php/revlatinofamilia/article/view/2272.

Morales, S. M. (2015). La familia y su evolución. Perfiles de las Ciencias Sociales, 3(5), 127-155. http://148.236.18.55/handle/20.500.12107/2557.

Oliva, E. y Villa, V. J. (2014). Hacia un concepto interdisciplinario de la familia en la globalización. Justicia Juris, 10(1), 11-20. https://dialnet.unirioja.es/servlet/ articulo? codigo $=5995439$.

Santana, E. V. (2019). La construcción de la resiliencia familiar en la experiencia de la discapacidad: una posibilidad para generar procesos inclusivos. Sinéctica, (53), 1-23. https://doi.org/10.31391/s2007-7033(2019)0053-012.

Suárez, P. A. y Vélez, M. (2018). El papel de la familia en el desarrollo social del niño: una mirada desde la afectividad, la comunicación familiar y estilos de educación parental. Revista Psicoespacios, 12(20), 173-198. https://doi.org/10.25057/ issn.2145-2776.

Tapia-Rodríguez, A. (2021). La gestión emocional en niñas de entre 6 y 12 años frente al divorcio de sus padres. https://rei.iteso.mx/handle/11117/6517.

Valdés,A.A.,Carlos,E.A.,Urías,M.eIbarra,B.G.(2011).Efectos del divorcio delos padres en el desempeño académico y la conducta de los hijos. Enseñanza e Investigación en Psicología, 16(2), 295-308. https://www.redalyc.org/pdf/292/29222521006.pdf.

Villavicencio, C. y Villarroel, M. (2017). Comunicación afectiva en familias desligadas. Fides Et Ratio. Revista de Difusión Cultural y Científica de la Universidad La Salle en Bolivia, 13(13), 15-39. http://www.scielo.org.bo/pdf/rfer/v13n13/v13n13_a03. pdf. 